\title{
Hall Effects on Unsteady MHD Free Convective Flow Past an Accelerated Moving Vertical Plate with Viscous and Joule Dissipations
}

\author{
B. C. Sarkar \\ Department of Applied \\ Mathematics \\ Vidyasagar University \\ Midnapore 721 102, India
}

\author{
S. Das \\ Department of Mathematics \\ University of Gour Banga \\ Malda 732 103, India
}

\author{
R. N. Jana \\ Department of Applied \\ Mathematics \\ Vidyasagar University \\ Midnapore 721 102, India
}

\begin{abstract}
Hall effects on an unsteady MHD free convective flow of a viscous incompressible electrically conducting fluid past a uniformly accelerated vertical plate in the presence of a uniform transverse applied magnetic field have been investigated on taking viscous and Joule dissipations into account. The governing partial differential equations have been solved numerically by applying a Crank - Nicolson's type of implicit finite difference method with a tri-diagonal matrix manipulation and an iterative procedure. The variations of the fluid velocity components and temperature distribution are presented graphically. It is found that the fluid velocity components are significantly affected by Hall parameter. An increase in Eckert number leads to rise in the fluid velocity components and temperature distribution. Further, it is found that the magnitude of the shear stress components at the plate increase with an increase in either Hall parameter or Eckert number or Prandtl number. The rate of heat transfer at the plate decreases with an increase in either Eckert number or Prandtl number or time.
\end{abstract}

Key words: Hall currents, MHD free convective flow, Prandtl number, Grashof number, Eckert number and viscous and Joule dissipations.

\section{INTRODUCTION}

Magnetohydrodynamics is currently undergoing a period of great enlargement and differentiation of subject matter. It is important in the design of MHD generators and accelerators in geophysics, underground water storage system, soil sciences, astrophysics, nuclear power reactor, solar structures and so on. The mechanism of conduction in ionized gases in the presence of strong magnetic field is different from that in metallic substance. The electric current in ionized gases is generally carried by electrons, which undergo successive collisions with other charged or neutral particles. In the ionized gases the current is not proportional to the applied potential except when the field is very weak in an ionized gas where the density is low and the magnetic field is very strong, the conductivity normal to the magnetic field is reduced due to the free spiraling of electrons and ions about the magnetic lines of force before suffering collisions and a current is induced in a direction normal to both electric and magnetic fields. This phenomenon is called the Hall effect. The study of Hall effects on MHD free convective flows has important engineering applications in problems of magnetohydrodynamic generators and Hall accelerators as well as in flight magnetohydrodynamics. It is also important in the solar physics involved in the sunspot development, the solar cycle and the structure of magnetic stars. The effect of Hall currents on hydromagnetic flow near a porous plate has been studied by Pop[1, 2]. Gupta [3] has examined the effect of Hall currents on the steady magnetohydrodynamic flow of an electrically conducting fluid past an infinite porous flat plate. Oscillatory magnetohydrodynamic flow past a flat plate with Hall effects has been investigated by Datta and Jana [4]. The effect of Hall currents on hydromagnetic free convective flow near an accelerated porous plate has been studied by Hossain and Mohammad [5]. Pop and Watanabe [6] have described the Hall effects on magnetohydrodynamic free convection about a semiinfinite vertical flat plate. Effect of chemical and thermal diffusion with the Hall current on an unsteady hydromagnetic flow past an infinitely long vertical porous plate has been discussed by Acharya et al. [7]. Aboeldahab and Elbarbary [8] have analyzed the effect of Hall current on the magnetohydrodynamics free convective flow past a semiinfinite vertical plate with mass transfer. Takhar et al.[9] have investigated the unsteady free convective flow past an infinitely long vertical porous plate due to the combined effects of thermal and mass diffusion, magnetic field and Hall currents. Kinyanjui et al.[10] have studied the heat and mass transfer in unsteady free convective flow past an impulsively started infinite vertical porous plate with Hall current and radiation absorption. Hall effects on an MHD flow past a moving plate in a fluid have been studied by Takhar et al.[11]. Convective heat transfer from different geometries has received considerable attention in recent years owing to its importance in various technological applications such as fibre and granular insulation, electronic system cooling, cool combustors, oil extraction, thermal energy storage and flow through filtering devices, porous material regenerative heat exchangers. The book written by Bejan and Kraus [12] excellently describes the extent of the research information in this area. The viscous dissipation effects are important in geophysical flows and also in certain industrial operations and are usually characterized by the Eckert number. In the literature, extensive research work is available to examine the effect of natural convection on flow past a plate. Callahan and Manner [13] have first considered the transient free convective flow past a semi-infinite plate by explicit finite difference method. However, this analysis is not applicable for other fluids whose Prandtl number is different from unity. Soundalgekar and Ganesan [14] have analyzed transient free convective flow past a semi-infinite vertical flat plate, on taking into account mass transfer by an implicit finite difference method of Crank-Nicolson type. Das and Jana [15] have examined the heat and mass transfer effects on an unsteady MHD free convective flow past a moving vertical plate in a porous medium. In most of the studies mentioned above, viscous dissipation is neglected. Gebhart [16] has shown the importance of viscous dissipative heat in free convective flow in the case of isothermal and constant heat flux at the plate. Gebhart and Mollendorf [17] have considered the effects of viscous dissipation for external natural convective flow over a surface. Israel Cookey et al. [18] have investigated the influence of viscous dissipation and radiation on an unsteady MHD free convective flow past an infinitely long heated vertical plate in a 
porous medium with time dependent suction. Srihari et al. [19] have studied an MHD free convective flow of an incompressible viscous dissipative fluid in an infinitely long vertical oscillating plate with constant heat flux. Suneetha et al. [20] have analyzed the effects of viscous dissipation and thermal radiation on hydromagnetic free convective flow past an impulsively started vertical plate. Babu et al. [21] have studied the radiation and chemical reaction effects on an unsteady MHD convective flow past a vertical moving porous plate embedded in a porous medium with viscous dissipation. Kishore et al. [22] have analyzed the effects of thermal radiation and viscous dissipation on MHD heat and mass diffusion flow past an oscillating vertical plate embedded in a porous medium with variable surface conditions. Hall effects on an unsteady MHD free convective flow past an impulsively started porous plate with viscous and Joule's dissipation have been studied by Anjali Devi et al. [23].

In the present paper, we have studied the Hall effects on an unsteady MHD free convective flow of a viscous incompressible electrically conducting fluid past an accelerated moving vertical plate on taking viscous and Joule dissipations into account. The governing equations have been solved numerically using Crank-Nicolson's method. It is found that the primary velocity $u$ and the magnitude of the secondary velocity $v$ increase with an increase in either Hall parameter $m$ or Eckert number $E c$ or Grashof number $G r$. It is also found that the fluid temperature $\theta$ increases with an increase in either Hall parameter $m$ or Eckert number $E c$ or Grashof number $G r$ or Prandtl number $\mathrm{Pr}$. Further, it is found that the absolute values of the shear stresses $\tau_{x}$ and $\tau_{y}$ at the plate $\eta=0$ increase with an increase in either $m$ or $E c$. The rate of heat transfer $-\left(\frac{\partial \theta}{\partial \eta}\right)_{\eta=0}$ at the plate $\eta=0$ decreases with an increase in either Eckert number $E c$ or Prandtl number $\mathrm{Pr}$ or time $\tau$.

\section{FORMULATION OF THE PROBLEM AND ITS SOLUTIONS}

Consider the unsteady hydrodynamic flow of a viscous incompressible electrically conducting fluid past a uniformly accelerated vertical plate on taking viscous and Joule dissipations into account. Choose a Cartesian co-ordinates system such that the $x$-axis is taken along the vertical plate in an upward direction, $z$-axis is perpendicular to the plate and $y$-axis is taken normal to the $z x$-plane. At time $t \leq 0$, both the fluid and the plate are at rest with constant temperature $T_{\infty}$. At time $t>0$, the plate at $z=0$ starts to move in its own plane with a uniform velocity $c t, c(>0)$ being a constant and $T_{w}$ is the plate temperature. A uniform transverse magnetic field of strength $H_{0}$ is applied perpendicular to the plate [see Fig.1]. As the plate is infinitely long, the fluid velocity components and temperature distribution are functions of $z$ and $t$ only.

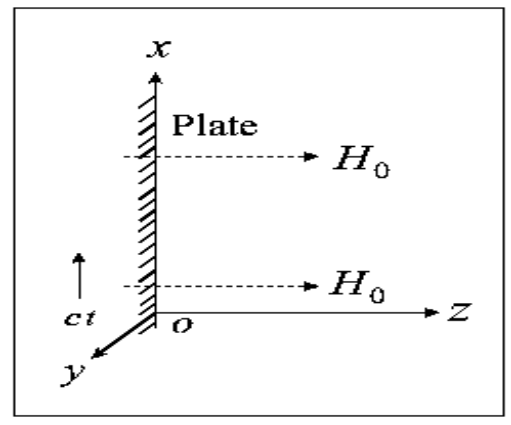

Figure 1. Geometry of the problem

The initial and boundary conditions of the problem are $u^{\prime}=v^{\prime}=0, T=T_{\infty}$ at $t \leq 0$ for all $z$,

$u^{\prime}=c t, v^{\prime}=0, T=T_{w}$ at $z=0$ for all $t>0$,

$u^{\prime} \rightarrow 0, v^{\prime} \rightarrow 0, T=T_{\infty}$ as $z \rightarrow \infty$ for all $t>0$,

where $u^{\prime}$ and $v^{\prime}$ are the fluid velocity components in the $x$ and $y$-directions respectively and $T$ the fluid temperature.

The generalized Ohm's law, on taking Hall currents into account and neglecting ion-slip and thermo-electric effect, is (see Cowling [24])

$$
\vec{J}+\frac{\omega_{e} \tau_{e}}{H_{0}}(\vec{J} \times \vec{H})=\sigma\left(\vec{E}+\mu_{e} \vec{q} \times \vec{H}\right),
$$

where $\vec{J}$ is the current density vector, $\vec{H}$ the magnetic field vector, $\vec{E}$ the electric field vector, $\omega_{e}$ the cyclotron frequency, $\tau_{e}$ the electron collision time, $\sigma$ the electrical conductivity of the fluid, $\mu_{e}$ the magnetic permeability and $\vec{q}$ the fluid velocity vector.

We shall assume that the magnetic Reynolds number for the flow is small so that the induced magnetic field can be neglected. This assumption is justified since the magnetic Reynolds number is generally very small for partially ionized gases. The solenoidal relation $\nabla \cdot \vec{H}=0$ for the magnetic field gives $H_{z}=H_{0}=$ constant everywhere in the fluid where $\vec{H} \equiv\left(0,0, H_{z}\right)$. Further, if $\left(J_{x}, J_{y}, J_{z}\right)$ be the components of the current density $\vec{J}$, then the equation of the conservation of the charge $\nabla \cdot \vec{J}=0$ gives $J_{z}=$ constant . This constant is zero since $J_{z}=0$ at the plate which is electrically non-conducting. Thus $J_{z}=0$ everywhere in the flow. Since the induced magnetic field is neglected, Maxwell's equation $\nabla \times \vec{E}=-\mu_{e} \frac{\partial \vec{H}}{\partial t}$ becomes $\nabla \times \vec{E}=\overrightarrow{0}$ which gives $\frac{\partial E_{x}}{\partial z}=0$ and $\frac{\partial E_{y}}{\partial z}=0$. This implies that $E_{x}=$ constant and $E_{y}=$ constant everywhere in the flow.

In view of the above assumption, the equation (2) gives

$$
\begin{aligned}
& J_{x}+m J_{y}=\sigma\left(E_{x}+\mu_{e} v^{\prime} H_{0}\right), \\
& J_{y}-m J_{x}=\sigma\left(E_{y}-\mu_{e} u^{\prime} H_{0}\right),
\end{aligned}
$$

where $m=\omega_{e} \tau_{e}$ is the Hall parameter.

At infinity, the magnetic field is uniform so that there is no current and hence, we have

$$
J_{x} \rightarrow 0, J_{y} \rightarrow 0 \text { as } z \rightarrow \infty .
$$

On the use of (1) and (5), equations (3) and (4) yield 


$$
E_{x}=0, E_{y}=0
$$

everywhere in the flow.

Substituting the values of $E_{x}$ and $E_{y}$ given by (6) in equations (3) and (4) and solving for $J_{x}$ and $J_{y}$, we get

$$
\begin{aligned}
& J_{x}=\frac{\sigma \mu_{e} H_{0}}{1+m^{2}}\left(v^{\prime}+m u^{\prime}\right), \\
& J_{y}=-\frac{\sigma \mu_{e} H_{0}}{1+m^{2}}\left(u^{\prime}-m v^{\prime}\right) .
\end{aligned}
$$

On the use of (7) and (8) and on using the usual Boussinesq approximation, the Navier-Stokes equations and energy equation can be written as

$$
\begin{aligned}
\frac{\partial u^{\prime}}{\partial t}= & v \frac{\partial^{2} u^{\prime}}{\partial z^{2}}+g \beta\left(T-T_{\infty}\right)-\frac{\sigma \mu_{e}^{2} H_{0}^{2}}{\rho\left(1+m^{2}\right)}\left(u^{\prime}-m v^{\prime}\right) \\
\frac{\partial v^{\prime}}{\partial t}= & v \frac{\partial^{2} v^{\prime}}{\partial z^{2}}-\frac{\sigma \mu_{e}^{2} H_{0}^{2}}{\rho\left(1+m^{2}\right)}\left(v^{\prime}+m u^{\prime}\right) \\
\rho c_{p} \frac{\partial T}{\partial t} & =k \frac{\partial^{2} T}{\partial z^{2}}+\mu\left[\left(\frac{\partial u^{\prime}}{\partial z}\right)^{2}+\left(\frac{\partial v^{\prime}}{\partial z}\right)^{2}\right] \\
& +\sigma \mu_{e}^{2} H_{0}^{2}\left(u^{\prime 2}+v^{\prime 2}\right),
\end{aligned}
$$

where $g$ is the acceleration due to gravity, $\beta$ the coefficient of thermal expansion, $\mu$ the coefficient of viscosity, $v$ the kinematic viscosity, $\rho$ the fluid density, $k$ the thermal conductivity and $c_{p}$ the specific heat at constant pressure.

Introducing the non-dimensional variables

$$
\begin{aligned}
& u=\frac{u^{\prime}}{(v c)^{\frac{1}{3}}}, v=\frac{v^{\prime}}{(v c)^{\frac{1}{3}}}, \eta=z\left(\frac{c}{v^{2}}\right)^{\frac{1}{3}}, \\
& \tau=t\left(\frac{c^{2}}{v}\right)^{\frac{1}{3}}, \theta=\frac{T-T_{\infty}}{T_{w}-T_{\infty}},
\end{aligned}
$$

equations (9), (10) and (11) become

$$
\begin{aligned}
\frac{\partial u}{\partial \tau} & =\frac{\partial^{2} u}{\partial \eta^{2}}+G r \theta-\frac{M^{2}}{\left(1+m^{2}\right)}(u-m v), \\
\frac{\partial v}{\partial \tau} & =\frac{\partial^{2} v}{\partial \eta^{2}}-\frac{M^{2}}{\left(1+m^{2}\right)}(v+m u), \\
\operatorname{Pr} \frac{\partial \theta}{\partial \tau} & =\frac{\partial^{2} \theta}{\partial \eta^{2}}+\operatorname{PrEc}\left[\left\{\left(\frac{\partial u}{\partial \eta}\right)^{2}+\left(\frac{\partial v}{\partial \eta}\right)^{2}\right\}\right. \\
+ & \left.M^{2}\left(u^{2}+v^{2}\right)\right]
\end{aligned}
$$

where $M^{2}=\frac{\sigma \mu_{e}^{2} H_{0}^{2}}{\rho}\left(\frac{v}{c^{2}}\right)^{\frac{1}{3}}$ is the magnetic parameter, $\operatorname{Pr}=\frac{\rho v c_{p}}{k}$ the Prandtl number, $G r=\frac{g \beta\left(T_{w}-T_{\infty)}\right.}{c}$ the Grashof number and $E c=\frac{(v c)^{\frac{2}{3}}}{c_{p}\left(T_{w}-T_{\infty}\right)}$ the Eckert number.

The corresponding initial and boundary conditions for $u(\eta, \tau)$, $v(\eta, \tau)$ and $\theta(\eta, \tau)$ are

$$
\begin{aligned}
& u=0, v=0, \theta=0 \text { for } \eta \geq 0 \text { and } \tau \leq 0, \\
& u=\tau, v=0, \theta=1 \text { at } \eta=0 \text { for } \tau>0, \\
& u \rightarrow 0, v \rightarrow 0, \theta \rightarrow 0 \text { as } \eta \rightarrow \infty \text { for } \tau>0 .
\end{aligned}
$$

\section{NUMERICAL SOLUTION}

Equations (13) - (15) are coupled, non-linear partial differential equations and these equations can not be solved exactly. However, these equations can be solved numerically. One of the most commonly used numerical methods is the finite difference technique which has better stability characteristics and is relatively simple, accurate and efficient. Another essential feature of this technique is that it is based on an iterative procedure and a tri-diagonal matrix manipulation. This method provides satisfactory results but it may fail when applied to problems in which the differential equations are very sensitive to the choice of initial conditions. In all numerical solutions the continuous partial differential equation is replaced with a discrete approximation. In this context the word discrete means that the numerical solution is known only at a finite number of points in the physical domain. The number of those points can be selected by the user of the numerical method. In general, increasing the number of points not only increases the resolution but also the accuracy of the numerical solution. The discrete approximation results are a set of algebraic equations that are evaluated (or solved) for the values of the discrete unknowns. The mesh is the set of locations where the discrete solution is computed. These points are called nodes and if one were to draw lines between adjacent nodes in the domain the resulting image would resemble a net or mesh.

When time dependent solutions are important, the CrankNicolson scheme has significant advantages. The CrankNicolson scheme is not significantly more difficult to implement and it has a temporal truncation error that is $O\left(\Delta \tau^{2}\right)$ as explained by Recktenwald [25]. The CrankNicolson scheme is implicit, it is also unconditionally stable $[26,27,28]$. In order to solve equations (13), (14) and (15) under the initial and boundary conditions (16), an implicit finite difference scheme of Crank-Nicolson's type has been employed. The right hand side of equations (13), (14) and (15) is approximated with the average of the central difference scheme evaluated at the current and the previous time step. The finite difference equations corresponding to equations (13), (14) and (15) are as follows

$$
\begin{aligned}
& \frac{u_{i, j+1}-u_{i, j}}{\Delta \tau}=\frac{1}{2(\Delta \eta)^{2}}\left[u_{i-1, j}-2 u_{i, j}+u_{i+1, j}\right. \\
& \left.+u_{i-1, j+1}-2 u_{i, j+1}+u_{i+1, j+1}\right] \\
& +\frac{G r}{2}\left(\theta_{i, j+1}+\theta_{i, j}\right)-\frac{M^{2}}{\left(1+m^{2}\right)}\left[\left(u_{i, j+1}+u_{i, j}\right)\right. \\
& \left.-m\left(v_{i, j+1}+v_{i, j}\right)\right] \text {, } \\
& \frac{v_{i, j+1}-v_{i, j}}{\Delta \tau}=\frac{1}{2(\Delta \eta)^{2}}\left[v_{i-1, j}-2 v_{i, j}+v_{i+1, j}\right. \\
& \left.+v_{i-1, j+1}-2 v_{i, j+1}+v_{i+1, j+1}\right] \\
& -\frac{M^{2}}{\left(1+m^{2}\right)}\left[\left(v_{i, j+1}+v_{i, j}\right)+m\left(u_{i, j+1}+u_{i, j}\right)\right], \\
& \operatorname{Pr} \frac{\theta_{i, j+1}-\theta_{i, j}}{\Delta \tau}=\frac{1}{2(\Delta \eta)^{2}}\left[\theta_{i-1, j}-2 \theta_{i, j}+\theta_{i+1, j}\right. \\
& \left.+\theta_{i-1, j+1}-2 \theta_{i, j+1}+\theta_{i+1, j+1}\right] \\
& +\operatorname{PrEc}\left[\left(\frac{u_{i+1, j}-u_{i, j}}{\Delta \eta}\right)^{2}+\left(\frac{v_{i+1, j}-v_{i, j}}{\Delta \eta}\right)^{2}\right.
\end{aligned}
$$




$$
\left.+M^{2}\left(u_{i, j}^{2}+v_{i, j}^{2}\right)\right]
$$

The initial and boundary conditions (16) become

$$
\begin{aligned}
& u_{i, 0}=0, v_{i, 0}=0, \theta_{i, 0}=0 \text { for all } i \neq 0, \\
& u_{0, j}=j \Delta \tau, v_{0, j}=0, \theta_{0, j}=1, \\
& u_{N, j}=0, v_{N, j}=0, \theta_{N, j}=0,
\end{aligned}
$$

where $N$ corresponds to $\infty$. Here the suffix $i$ corresponds to $\eta$ and $j$ corresponds to $\tau$. Also $\Delta \tau=\tau_{j+1}-\tau_{j}$ and $\Delta \eta=\eta_{i+1}-\eta_{i}$. Knowing the values of $\theta, u$ and $v$ at a time $\tau$ we can evaluate the values at a time $\tau+\Delta \tau$ as follows. We substitute $i=1,2, \ldots, N-1$ in the equation (19) which constitute a tri-diagonal system of equations, the system can be solved by Thomas algorithm as discussed by Carnahan et al.[29]. Thus $\theta$ is known for all values of $\eta$ at time $\tau$. Then knowing the values of $\theta$ and applying the same procedure with the boundary conditions, we calculate $u$ and $v$ from equations (17) and (18). This procedure is continued to obtain the solution till desired time $\tau$. The Crank-Nicolson scheme has a truncation error of $O\left(\Delta \tau^{2}\right)+O\left(\Delta \eta^{2}\right)$, i.e. the temporal truncation error is significantly small.

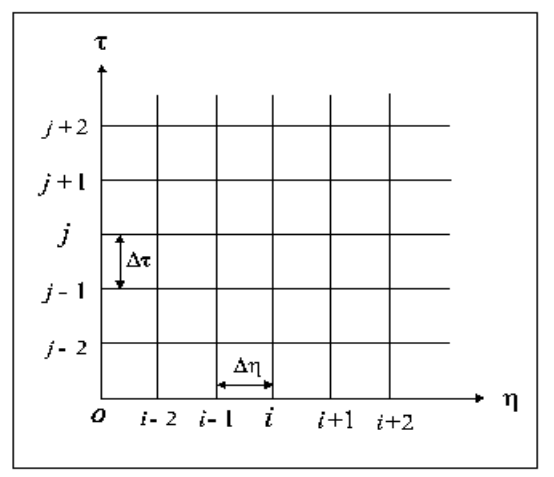

\section{Figure 2. Finite difference grids}

The implicit method gives stable solutions and requires matrix inversions which we have done at step forward in time because this problem is an initial-boundary value problem with a finite number of spatial grid points, though the corresponding difference equations do not automatically guarantee the convergence of the mesh $\Delta \eta \rightarrow 0$. To achieve maximum numerical efficiency, we have used the tri-diagonal procedure to solve the two-point conditions governing the main coupled governing equations of momentum and energy. The convergence (consistency) of the process is quite satisfactory and the numerical stability of the method is guaranteed by the implicit nature of the numerical scheme. Hence, the scheme is consistent. The stability and consistency ensure convergence.

\section{RESULTS AND DISCUSSION}

We have presented the non-dimensional fluid velocity components $u, v$ and the fluid temperature distribution $\theta$ for several values of the magnetic parameter $M^{2}$, Hall parameter $m$, Prandtl number $\mathrm{Pr}$, Grashof number $\mathrm{Gr}$, Eckert number $E c$ and time $\tau$ against $\eta$ in Figs.3-19. It is seen from Figs.3 and 4 that the primary velocity $u$ decreases and the magnitude of the secondary velocity $v$ increases with an increase in magnetic parameter $M^{2}$. This indicates that the applied magnetic field is effectively moving with the fluid motion. The application of the transverse magnetic field plays the role of a resistive type force (Lorentz force) on the primary flow similar to a drag force (that acts in the opposite direction of the fluid motion), which tends to resist the flow thereby reducing the primary velocity. On the other hand, the resulting Lorentzian body force will not act as a drag force but act as an aiding body force on the secondary flow. This will serve to accelerate the secondary velocity. It is observed from Figs. 5 and 6 that the primary velocity $u$ and the magnitude of the secondary velocity $v$ increase with an increase in Hall parameter $m$. This is because, in general, the Hall currents reduce the resistance offered by the Lorentz force. This means that Hall currents have a tendency to increase the fluid velocity components. Figs.7 and 8 show that the primary velocity $u$ and the magnitude of the secondary velocity $v$ increase with an increase in Grashof number $G r$. Grashof number $G r$ signifies the relative effect of the thermal buoyancy force to the viscous hydrodynamic force. As expected, it is observed that there is a rise in the fluid velocity due to the enhancement of thermal buoyancy force. It is due to the fact that an increase of Grashof number has a tendency to increase the thermal effect. It is observed from Figs. 9 and 10 that the primary velocity $u$ and the magnitude of the secondary velocity $v$ decrease with an increase in Prandtl number $P r$. Physically this is due to the fact that fluids with high Prandtl number have greater viscosity, which makes the fluid thick and hence move slowly. Figs.11 and 12 show that the primary velocity $u$ and the magnitude of the secondary velocity $v$ increase with an increase in Eckert number $E c$. Figs.13 and 14 display that the primary velocity $u$ and the magnitude of the secondary velocity $v$ increase with an increase in time $\tau$. Figs.15-19 show that the fluid temperature $\theta$ increases with an increase in either magnetic parameter $M^{2}$ or Hall parameter $m$ or Grashof number $G r$ or Prandtl number $P r$ or Eckert number $E c$. Eckert number is the ratio of the kinetic energy of the flow to the boundary layer enthalpy difference. The effect of viscous dissipation on the flow field is to increase the energy, yielding a greater fluid temperature and as a consequence greater buoyancy force. The increase in the buoyancy force due to an increase in the dissipation parameter enhances the temperature ditribution. It is seen from Figs.15-19 that the maximum temperature occur in the vicinity of the plate and asymptotically approaches to zero in the free stream region. This is because the effect of the magnetic parameter and Hall parameter can be felt only in the Hartmann layer and the thermal boundary layer respectively. It can be concluded that the fluid velocity and temperature raise due to viscous and Joule dissipations.

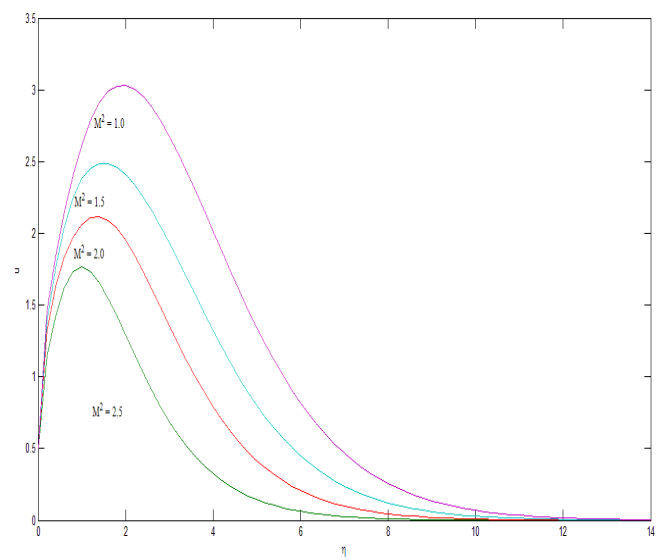


Figure 3. Primary velocity for $M^{2}$ when $m=0.5$, $G r=5, E c=0.5, P r=0.25$ and $\tau=0.2$

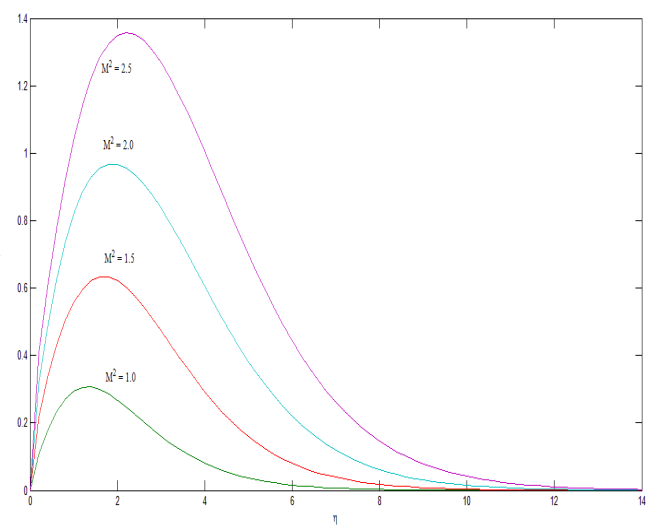

Figure 4. Secondary velocity for $M^{2}$ when $m=0.5$, $G r=5, E c=0.5, P r=0.25$ and $\tau=0.2$

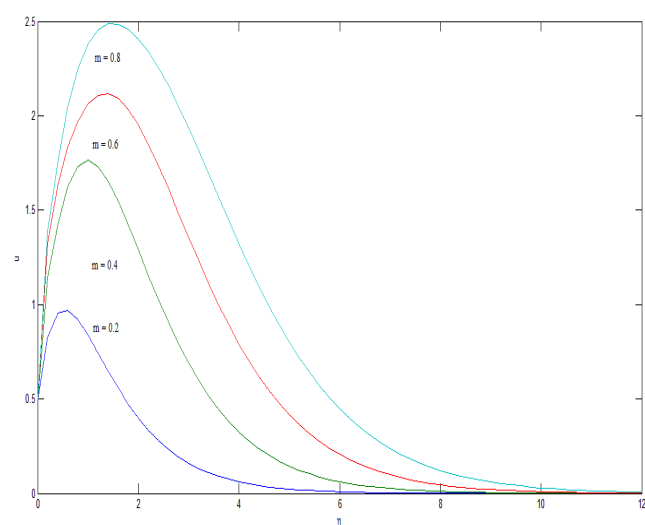

Figure 5. Primary velocity for $m$ when $M^{2}=2, G r=5$, $E c=0.5, \operatorname{Pr}=0.25$ and $\tau=0.2$

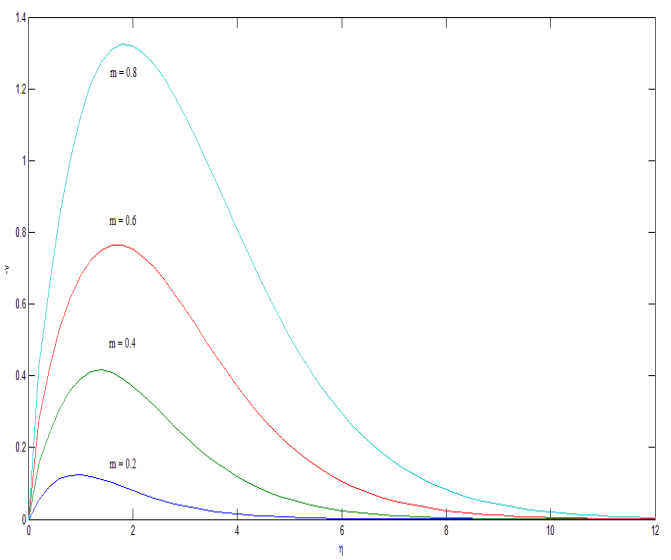

Figure 6. Secondary velocity for $m$ when $M^{2}=2$, $G r=5, E c=0.5, \operatorname{Pr}=0.25$ and $\tau=0.2$

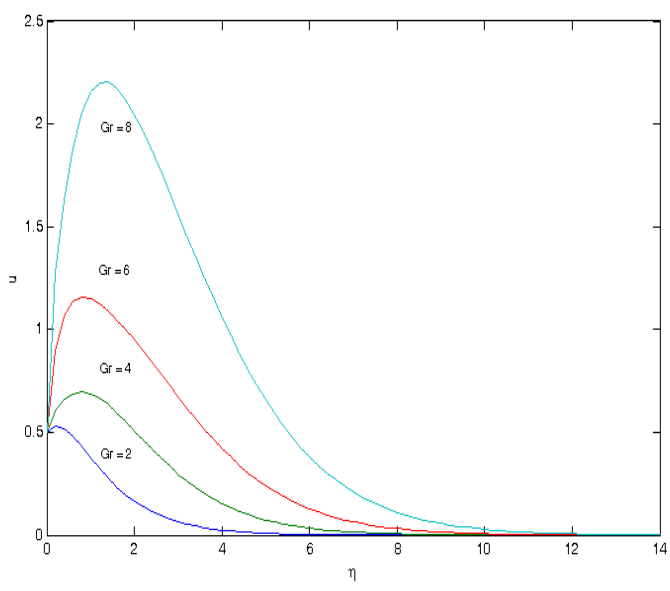

Figure 7. Primary velocity for $G r$ when $M^{2}=2$, $m=0.5, E c=0.5, P r=0.25$ and $\tau=0.2$

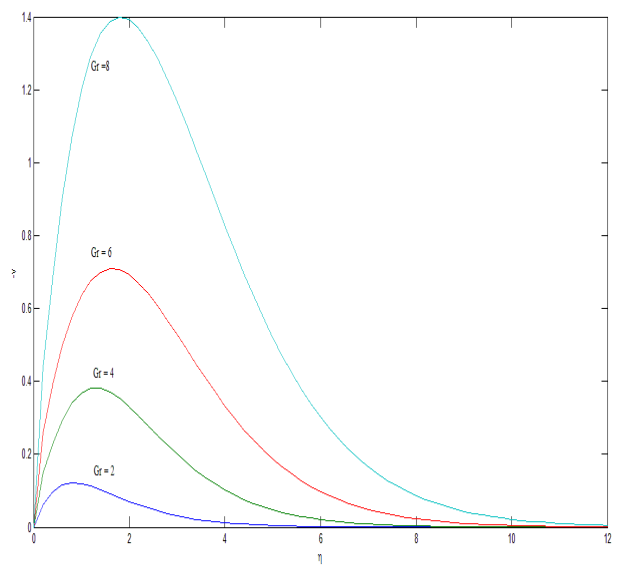

Figure 8. Secondary velocity for $G r$ when $M^{2}=2$, $m=0.5, E c=0.5, \operatorname{Pr}=0.25$ and $\tau=0.2$

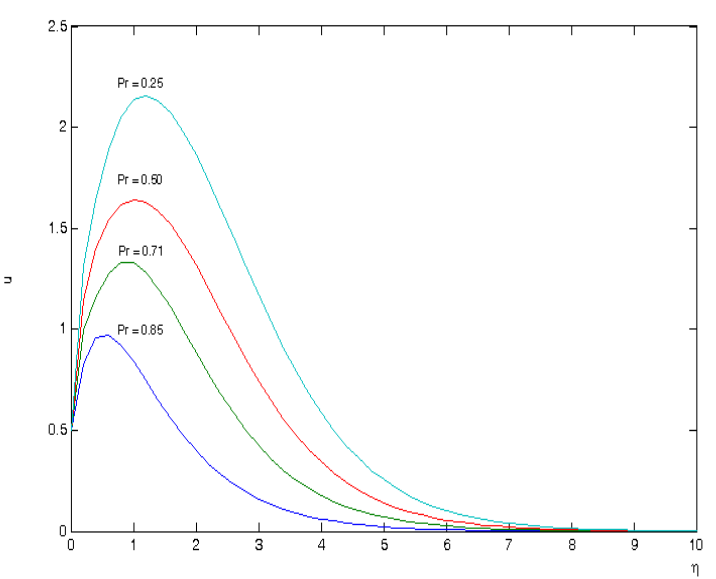

Figure 9. Primary velocity for $\operatorname{Pr}$ when $M^{2}=2$, $m=0.5, E c=0.5, G r=5$ and $\tau=0.2$ 


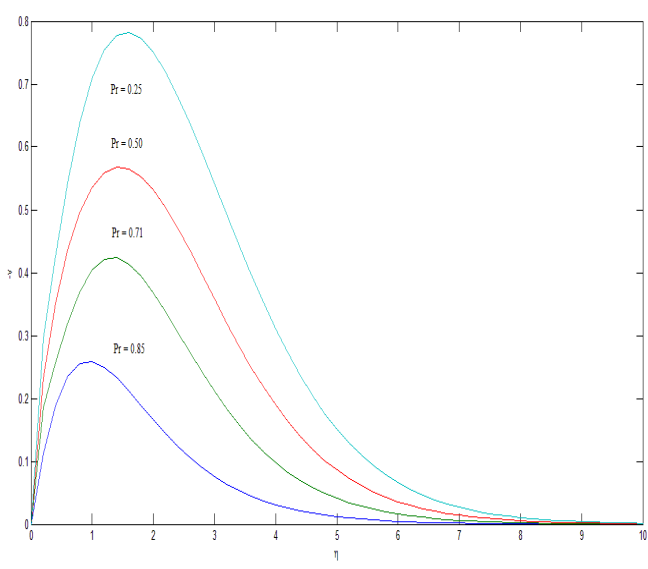

Figure 10. Secondary velocity for $\operatorname{Pr}$ when $M^{2}=2$, $m=0.5, E c=0.5, G r=5$ and $\tau=0.2$

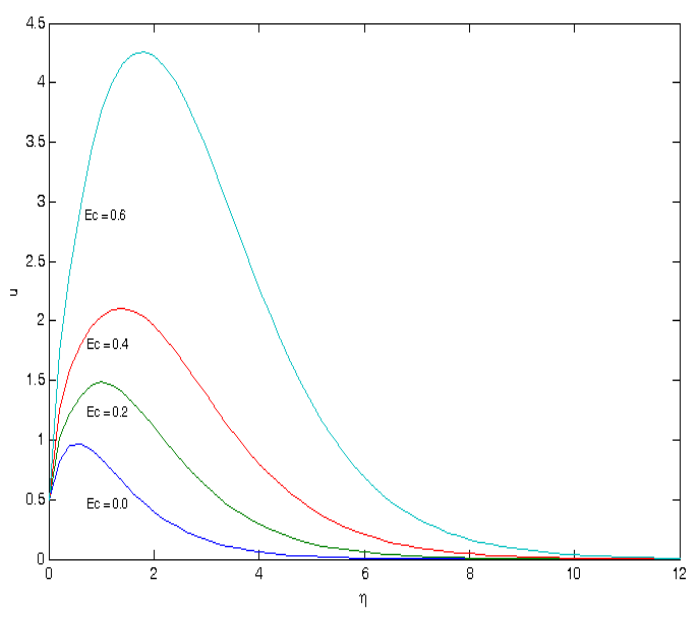

Figure 11. Primary velocity for $E c$ when $M^{2}=2$, $m=0.5, P r=0.25, G r=5$ and $\tau=0.2$

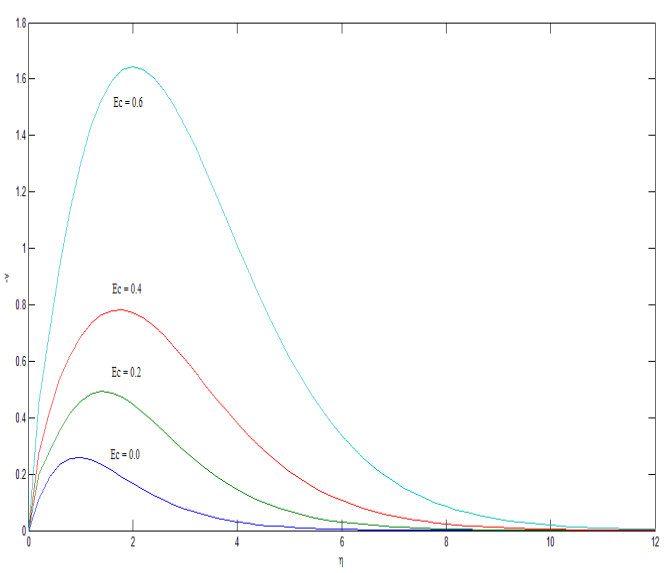

Figure 12. Secondary velocity for $E c$ when $M^{2}=2$, $m=0.5, P r=0.25, G r=5$ and $\tau=0.2$

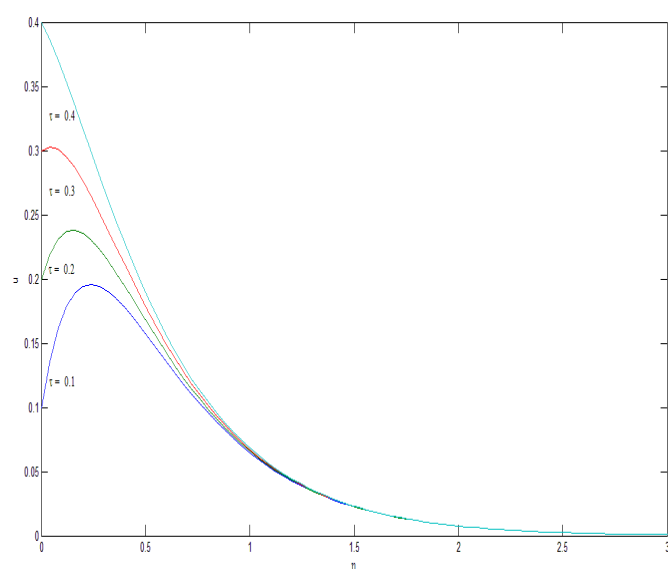

Figure 13. Primary velocity for $\tau$ when $M^{2}=2$, $m=0.5, P r=0.25, G r=5$ and $E c=0.5$

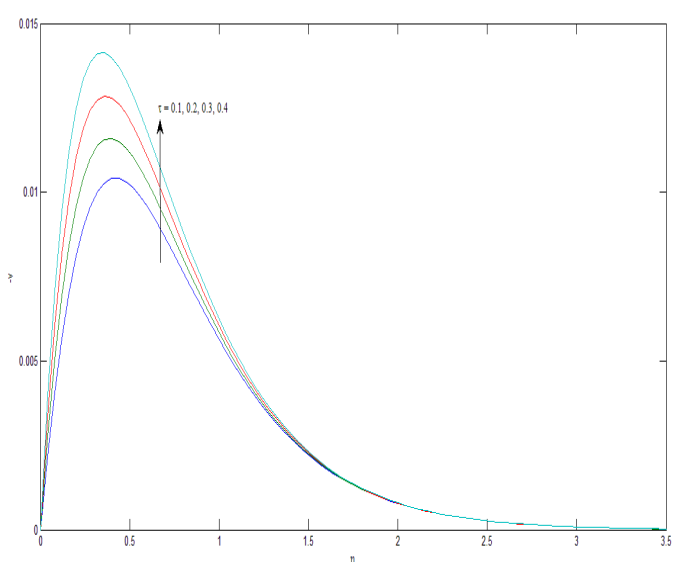

Figure 14. Secondary velocity for $\tau$ when $M^{2}=2$, $m=0.5, \operatorname{Pr}=0.25, G r=5$ and $E c=0.5$

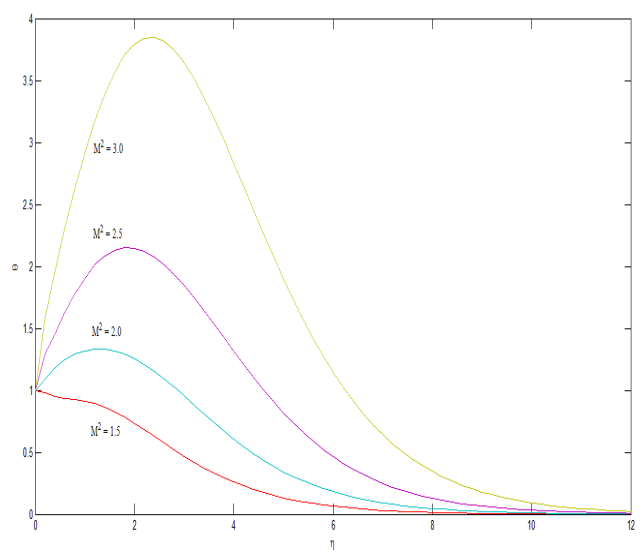

Figure 15. Temperature for $M^{2}$ when $P r=0.25$, $m=0.5, G=5, E c=0.5$ and $\tau=0.2$ 


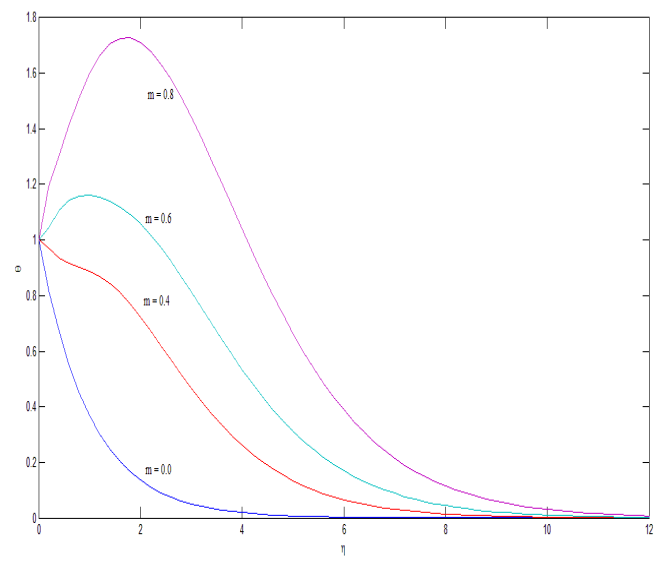

Figure 16. Temperature for $m$ when $\operatorname{Pr}=0.25, M^{2}=2$, $G=5, E c=0.5$ and $\tau=0.2$

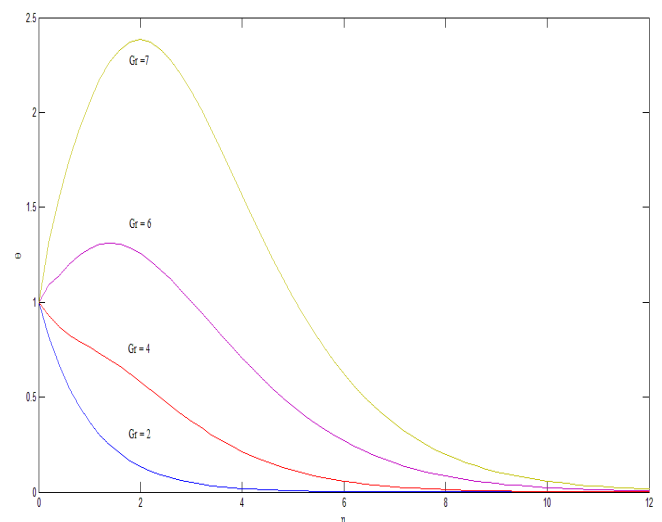

Figure 17. Temperature for $G r$ when $P r=0.25$, $m=0.5, M^{2}=2, E c=0.5$ and $\tau=0.2$

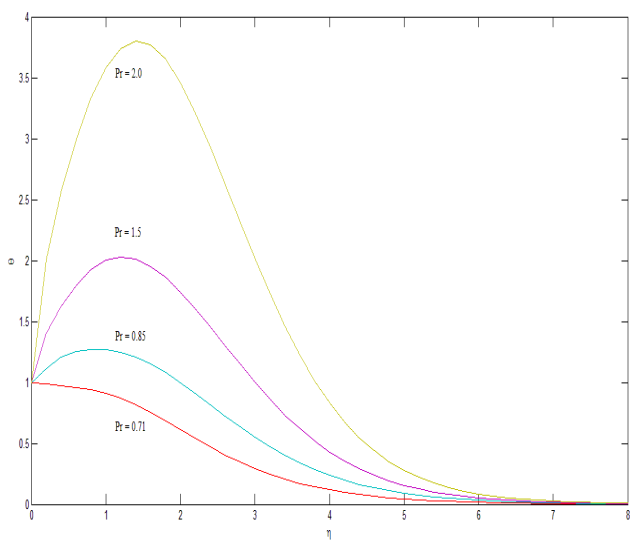

Figure 18. Temperature for $\operatorname{Pr}$ when $G r=5, m=0.5$, $M^{2}=2, E c=0.5$ and $\tau=0.2$

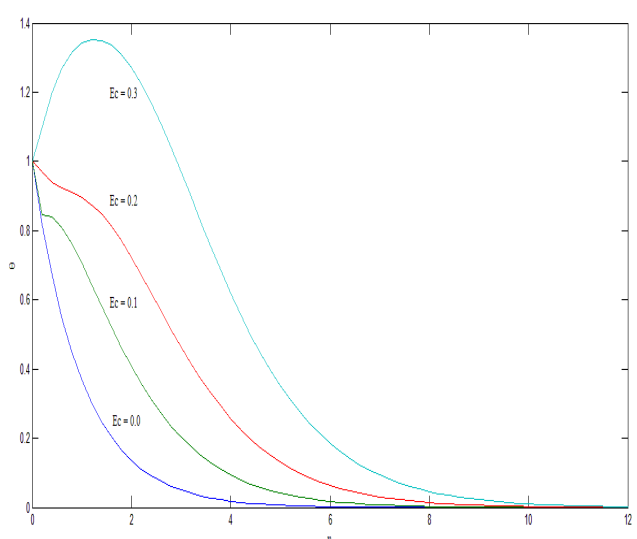

Figure 19. Temperature for $E c$ when $\operatorname{Pr}=0.25$, $m=0.5, M^{2}=2, G r=5$ and $\tau=0.2$

Numerical values of the rate of heat transfer $-\left(\frac{\partial \theta}{\partial \eta}\right)_{\eta=0}$ at the plate $\eta=0$ are presented in Table 1 for several values of Eckert number $E c$, Prandtl number $\operatorname{Pr}$ and time $\tau$. It is seen from Table 1 that the rate of heat transfer $-\left(\frac{\partial \theta}{\partial \eta}\right)_{\eta=0}$ at the plate $\eta=0$ decreases with an increase in either Prandtl number $\operatorname{Pr}$ or time $\tau$ or Eckert number $E c$. The negative values of the rate of heat transfer show that the heat is transferred from the plate to fluid. 
Table 1. Rate of heat transfer $-\left(\frac{\partial \theta}{\partial \eta}\right)_{\eta=0}$ at the plate $\eta=0$

\begin{tabular}{|c|c|c|c|c|c|c|c|c|}
\hline \multicolumn{5}{|c|}{$P r$} & \multicolumn{4}{|c|}{$\tau$} \\
\hline$E c$ & 0.25 & 0.5 & 0.71 & 0.85 & 0.2 & 0.4 & 0.6 & 0.8 \\
\hline 0.1 & 0.76623 & 0.51754 & 0.43898 & 0.28913 & 0.76623 & 0.66217 & 0.57816 & 0.56610 \\
\hline 0.2 & 0.65748 & 0.49519 & 0.42438 & 0.27528 & 0.65748 & 0.56210 & 0.43391 & 0.35619 \\
\hline 0.3 & 0.58738 & 0.46857 & 0.39607 & 0.23467 & 0.58738 & 0.50352 & 0.47549 & 0.32894 \\
\hline 0.4 & 0.53618 & 0.43675 & 0.35442 & 0.16665 & 0.53618 & 0.45019 & 0.34108 & 0.20002 \\
\hline
\end{tabular}

The non-dimensional shear stresses $\tau_{x}$ and $\tau_{y}$ at the plate $\eta=0$ due to the primary and secondary flows are respectively given by

$$
\tau_{x}=\left(\frac{\partial u}{\partial \eta}\right)_{\eta=0} \text { and } \tau_{y}=\left(\frac{\partial v}{\partial \eta}\right)_{\eta=0} .
$$

Numerical values of the non-dimensional shear stresses $\tau_{x}$ and $\tau_{y}$ at the plate $\eta=0$ are presented in Figs.20-24 against magnetic parameter $M^{2}$ for several values of Hall parameter $m$, Grashof number $\mathrm{Gr}$, Prandtl number $\mathrm{Pr}$, Eckert number $E c$ and time $\tau$. Figs.20-23 show that the shear stress $\tau_{x}$ and the absolute value of the shear stress $\tau_{y}$ at the plate $\eta=0$ increase with an increase in either Hall parameter $m$ or Grashof number $\mathrm{Gr}$ or Prandtl number $\mathrm{Pr}$ or Eckert number $E c$. Fig. 24 shows that the shear stress $\tau_{x}$ at the plate $\eta=0$ increases while the absolute value of the shear stress $\tau_{y}$ at the plate $\eta=0$ decreases with an increase in time $\tau$. On the other hand, it is observed from Figs.20-24 that the shear stress $\tau_{x}$ and the absolute value of the shear stress $\tau_{y}$ at the plate $\eta=0$ at first increase, reach a maximum and then decrease with an increase in magnetic parameter $M^{2}$.

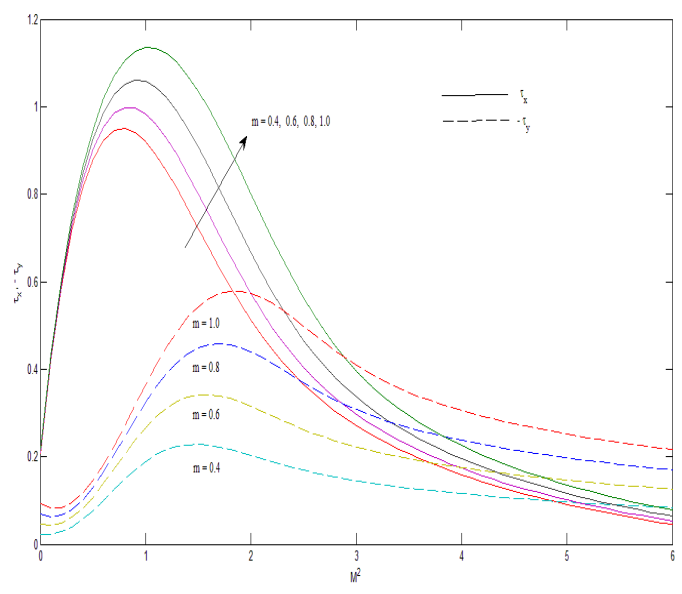

Figure 20. Shear stress $\tau_{x}$ and $\tau_{y}$ for $m$ when $\operatorname{Pr}=0.25, G r=5, E c=0.5$ and $\tau=0.2$

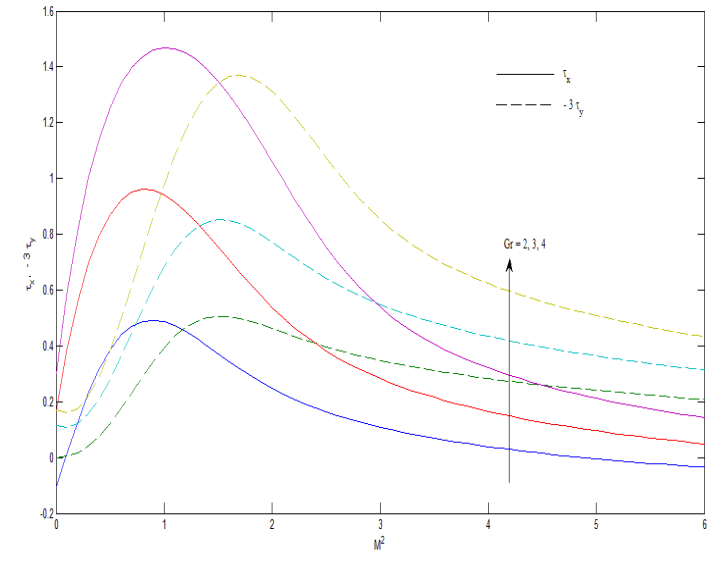

Figure 21. Shear stress $\tau_{x}$ and $\tau_{y}$ for $G r$ when $\operatorname{Pr}=0.25, m=0.5, E c=0.5$ and $\tau=0.2$

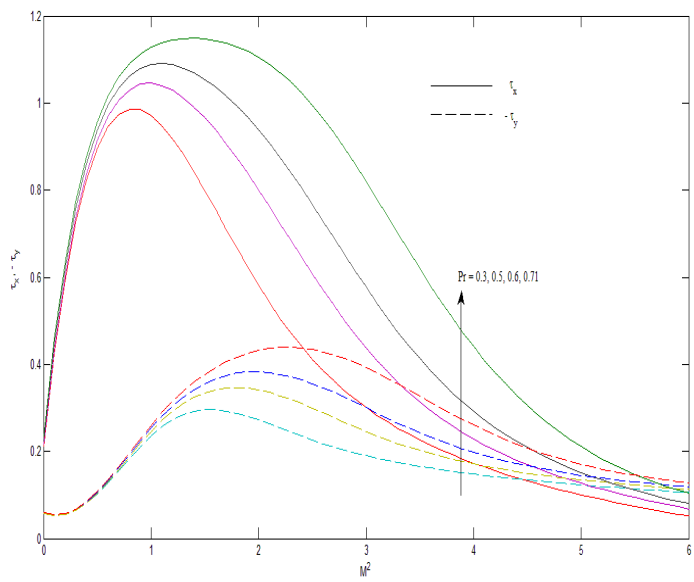

Figure 22. Shear stress $\tau_{x}$ and $\tau_{y}$ for $\operatorname{Pr}$ when $m=0.5$, $G r=5, E c=0.5$ and $\tau=0.2$ 


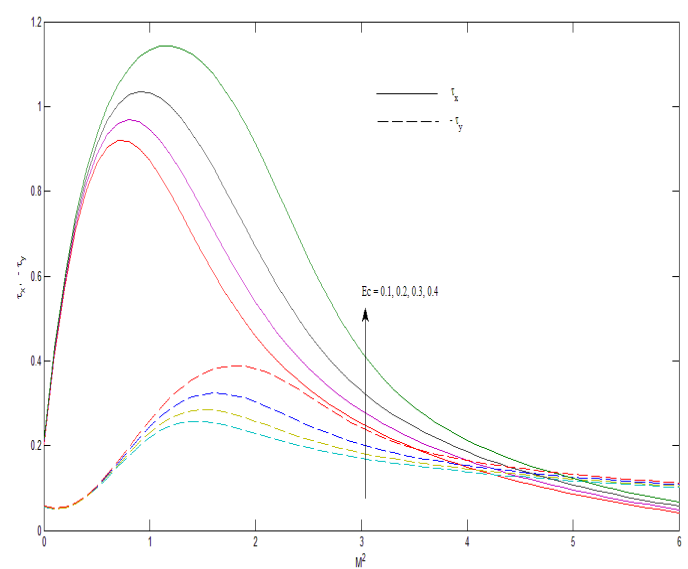

Figure 23. Shear stress $\tau_{x}$ and $\tau_{y}$ for $E c$ when $P r=0.25, G r=5, m=0.5$ and $\tau=0.2$

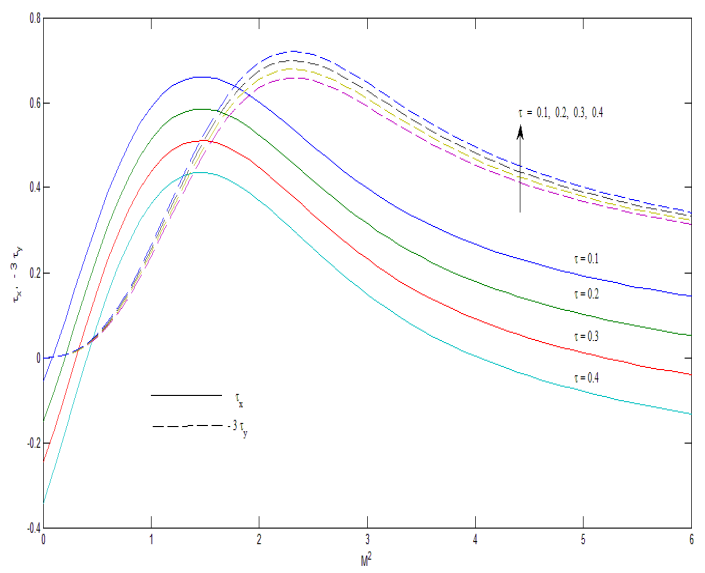

Figure 24. Shear stress $\tau_{x}$ and $\tau_{y}$ for time $\tau$ when $P r=0.25, G r=5, E c=0.5$ and $m=0.5$

\section{CONCLUSION}

Hall effects on an unsteady MHD free convective flow of a viscous incompressible electrically conducting fluid past an accelerated vertical flat plate have been studied on taking viscous and Joule dissipations into account. It is observed that the Hall currents accelerate the fluid velocity components. The fluid velocity and temperature raise due to viscous and Joule dissipations. Further, it is found that the shear stress $\tau_{x}$ and the absolute value of the shear stress $\tau_{y}$ at the plate increase with an increase in either Hall parameter or Prandlt number or Eckert number. The rate of heat transfer at the plate decreases with an increase in either Prandtl number or Eckert number or time.

\section{REFERENCES}

[1] Pop, I. (1971). The effect of Hall currents on hydromagneticflow near an accelerated plate. J. Math. Phys. Sci. 5: 375-379.

[2] Pop, I. (1974). Effects of Hall currents on hydromagnetic flow near a porous plate. Acta Mechanica. 20: 316-318.

[3] Gupta, A.S. (1975). Hydromagnetic flow past a porous flat plate with Hall effects. Acta Mechanica. 22: 281-267.
[4] Datta, N. and Jana, R. N. (1976). Oscillatory magnetohydrodynanic flow past a flat plate with Hall effects. J. Phys. Soc. Japn. 40: 1469-1474.

[5] Hossain, M. A. and Mohammad, K. (1988). Effect of Hall currents on hydromagnetic free convection flow near an accelerated porous plate. Jpn. J. Appl. Phys. 27(8): 15311535 .

[6] Pop, I. and Watanabe, T. (1994). Hall effects on magnetohydrodynamic free convection about a semiinfinite vertical flat plate. Int. J. Eng. Sci. 32: 1903-1911.

[7] Acharya, M., Dash, G. C. and Singh, L. P. (1995). Effect of chemical and thermal difiusion with Hall current on unsteady hydromagnetic flow near an infinite vertical porous plate. J. Phys. D: Appl. Phys. 28: 2455-2464.

[8] Aboeldahab, E. M. and Elbarbary, E. M. E. (2001). Hall current effect magnetohydrodynamics free convection flow past a semi-infinite vertical plate with mass transfer. Int. J. Eng. Sci. 39: 1641- 1652.

[9] Takhar, H. S., Roy, S. and Nath, G. (2003). Unsteady free convection flow over an infinite vertical porous plate due to the combined effects of thermal and mass difiusion, magnetic field and Hall currents. Heat and Mass Trans. 39: $825-834$.

[10] Kinyanjui, M., Kwanja, J. K. and Uppal, S. M. (2001). Magnetohydrodynamics free convection heat and mass transfer of a heat generating fluid past an impulsively started inifinite vertical porous plate with Hall Current and radiation absorption. Energy Conversion and Manegment. 42: 917-931.

[11] Takhar, H. S., Chamkha, A. J. and Nath, G. (2002). MHD flow over a moving plate in a rotating fluid with magnetic field, Hall currents and free-stream velocity. Int. J. Eng. Sci. 40: 1511-1527.

[12] Bejan, A. and Kraus, A. D., Heat Transfer Handbook, Wiley, New York, 2003.

[13] Callahan, G. D. and Manner, W. J. (1976). Transient free convection with mass transfer on an isothermal vertical flat plate. Int. J. Heat Mass Trans. 19: 165-174.

[14] Soundalgekar, V. M. and Ganesan, P. (1981). Finite difference analysis of transient free convection with mass transfer on an isothermal vertical flat plate. Int. J. Eng. Sci. 19: 757-770.

[15] Das, K. and Jana, S. (2010). Heat and Mass transfer effects on unsteady MHD free convection flow near a moving vertical plate in a porous medium. Bull. Soc. Math. 17: 1532 .

[16] Gebhart, B. (1962). Effect of viscous dissipation in natural convection. J. Flu. Mech. 14(2): 225-232.

[17] Gebhart, B. and Mollendraf, J. (1969). Viscous dissipation in external natural convection flows. J. Flu. Mech. 38: 97107.

[18] Israel-Cookey, C., Ogulu, A. and Omubo-Pepple, V. B. (2003). Influence of viscous dissipation on unsteady MHD free convection flow past an infinite heated vertical plate in porous medium with time-dependent suction. Int. J. Heat mass Trans. 46: 2305-2311.

[19] Srihari, K., Anand Rao, J. and Kishan, N. (2006). MHD 
free convection flow of an incompressible viscous dissipative fluid in an infinite vertical oscillating plate with constant heat flux. J. Energy, Heat and Mass Trans. 28: $19-28$.

[20] Suneetha, S., Bhaskar Reddy, N. and Ramachandra Prasad, V. (2008). Effects of viscous dissipation and thermal radiation on hydromagnetic free convection flow past an impulsively started vertical plate. J. Naval Archit. Marine Eng. 2: 57-70.

[21] Sudheer Babu, M., Satya Narayana, P. V., Sankar Reddy, T. and Umamaheswara Reddy, D. (2011). Radiation and chemical reaction effects on an unsteady MHD convection flow past a vertical moving porous plate embedded in a porous medium with viscous dissipation. Adv. Appl. Sci. Res. 2(5): 226-239 .

[22] Kishore, P. M., Rajesh, V. and Vijayakumar Verma, S. (2012). The effects of thermal radiation and viscous dissipation on MHD heat and mass diffusion flow past an oscillating vertical plate embedded in a porous medium with variable surface conditions. Theoret. Appl. Mech. 39(2) : 99-125.
[23] Anjali Devi, S. P., Shailendhra, K., Ramesan, C. V. (2012). Hall effect on unsteady MHD free convection flow past an impulsively started porous plate with viscous and Joule's dissipation. Int. J. Sci. and Eng. Investigations. 1(6): 64-71

[24] Cowling, T. G. (1957). Magnetohydrodynamics, Interscience Publisher, Inc, New York.

[25] Recktenwald, G. W. (2011). Finite-difference approximations to the heat equation.

[26] Ames, W.F. (1992). Numerical Methods for Partial Differential Equations, Academic Press, Inc., Boston, Third edition.

[27] Isaacson, E. and Keller, H. B. (1994). Analysis of Numerical Methods, Dover, New York.

[28] Burden, R. L. and Faires, J. D. (1997). Numerical Analysis, Brooks/Cole Publishing Co., New York, Sixth edition.

[29] Carnahan, B., Luther, H. A. and Wilkes, J. O. (1969). Appl. Numerical Meth., John Wiley \& Sons, New York. 